\title{
GLASGOW UNIVERSITY RADIOCARBON MEASUREMENTS IX
}

\author{
J A CAMPBELL* and M S BAXTER \\ Department of Chemistry, University of Glasgow, G12 8QQ

\section{INTRODUCTION}

The following date list presents results obtained during 1975-1977. The facilities at Glasgow have been further developed and include a gas counting system utilizing $\mathrm{CO}_{2}$ as counting material and two liquid scintillation counting laboratories based on synthesis of benzene. The results presented here were obtained using the liquid scintillation system only. Sample pretreatment varied according to individual sample type, whether wood or charcoal. The majority of samples were of preserved wood. After manual removal of gross contamination, samples of wood were finely chopped and digested in boiling, 2M KOH solution. The dehumified wood was then separated by filtration, thoroughly washed with distilled water and dilute hydrochloric acid, and then bleached in a solution of $\mathrm{NaClO}_{2} / \mathrm{HCl}$ at $80^{\circ} \mathrm{C}$ for 48 hours. Pure, white wood cellulose was obtained by filtration and washed with a large volume of distilled water. The cellulose, typically $40 \%$ by weight of the original material, was then dried overnight in an oven at $80^{\circ} \mathrm{C}$. Samples of charcoal were closely examined for non-contemporaneous contamination and then subjected to successive treatments with hot, dilute (1M) $\mathrm{KOH}$ and $\mathrm{HCl}$, respectively. The remaining charcoal was then dried overnight at $80^{\circ} \mathrm{C}$.

Pretreated samples were converted to benzene for counting using standard procedures. In summary, samples were converted to $\mathrm{CO}_{2}$ by combustion in a stainless steel pressure reaction vessel. A sample of $\mathrm{CO}_{2}$ was removed for subsequent mass spectrometric assay. $\mathrm{CO}_{2}$ was converted to $\mathrm{C}_{2} \mathrm{H}_{2}$ by reaction with molten lithium in a second stainless steel reaction vessel. When cool, the product lithium carbide was hydrolyzed with freshly distilled water, yielding acetylene. Finally benzene was produced by cyclotrimerization over an activated catalyst (KC Perl Katalysator Neu).

Samples were counted using a Packard Tricarb Series 3330 Liquid Scintillation Spectrophotometer optimized for ${ }^{14} \mathrm{C}$ detection. A standard $10 \mathrm{ml}$ counting geometry was adopted, the scintillation cocktail comprising $8 \mathrm{ml}$ synthesized benzene and $2 \mathrm{ml}$ of a PPO/POPOP toluene-based scintillation solution. (Where insufficient benzene was synthesized, samples were diluted to the standard volume with "dead" spectroscopic grade benzene.) The long-term stability of the counting system was assessed by continuous monitoring of background and NBS oxalic acid modern standard samples. An excellent degree of stability was indicated. Count rates for NBS oxalic acid and background samples were measured as $63.40 \pm$ $0.04 \mathrm{cpm}$ and $7.44 \pm 0.01 \mathrm{cpm}$, respectively. Observed sample and standard activities were corrected for the effects of quenching by means of a sample channels ratio method. Measurement of the stable carbon isotope

* Present address: Atlantic Oceanographic Laboratory, Bedford Institute of Oceanography, Dartmouth, Nova Scotia, B2Y 4A2 Canada 
enrichment, $\delta^{13} \mathrm{C}$, was performed using a VG Micromass 602B stable isotope mass spectrometer to a precision of $\pm 0.05 \%$ co $( \pm 1 \sigma)$. The uncertainty quoted on radiocarbon age determinations reported here represents the $\pm 1 \sigma$ counting errors alone and does not include contributions from extraneous experimental variability.

\section{ACKNOWLEDGMENTS}

We wish to thank A Heyworth for supplies of dendrochronologically dated wood. The participation of N Drndarski, M J Stenhouse, and D D Harkness in intercalibration exercises is also acknowledged. J A C is grateful to the Natural Environment Research Council for generous financial support. The competent technical assistance of $\mathrm{K}$ McKay and A Miller is acknowledged.

\section{SAMPLE DESCRIPTIONS}

\section{INTERCALIBRATION SAMPLES}

Intercalibration samples were analyzed at regular intervals during 1975-1977. In addition to samples subm specifically for intercalibration purposes, a number of "check" analyses was performed on selected treering samples. Results of check analyses will be reported with appropriate tables in subsequent secs of the text. (The designation "GUA" refers to analyses on the Glasgow $\mathrm{CO}_{2}$ gas counting system which have yet to be assigned GU- code numbers.)

GU.656.

$166 \pm 42$

Hitchcock wood. Comment: LJ-3451, $142 \pm 68$; LJ-3452, $221 \pm 89$.

GU-657.

$$
\Delta^{14} \mathbf{C}=\mathbf{5 . 0 1} \pm \mathbf{5 . 6 2} \% \circ
$$

Charcoal. Comment: LJ-3448, $\Delta^{14} \mathrm{C}=-3.8 \pm 6.1 \%$.

GU-658.

$3505 \pm 58$

Pine wood. Comment: LJ-3453, $3793 \pm 61$; GUA, $3551 \pm 58$.

GU-659. Penzance, Cornwall

$4093 \pm 50$

Wood. Comment: sample previously described and reported under SRR-714, $4278 \pm 50$. Also GUA, $4025 \pm 67$.

GU-660. West Runton 6

$26,450 \pm 420$

Wood. Comment: sample previously described and reported under SRR-225, -228, >47,500; GrN-6819, >54,200; GrN-6892, >47,400. Also GUA, $21,732 \pm 532$.

\section{GU-661. West Runton 6}

$$
26,400 \pm 480
$$

\section{As GU-660.}

General Comment: discrepancy between age determinations on West Runton 6 sample for Glasgow systems, both liquid scintillation and gas counting, and those at SRR and GrN, suggests sample contamination (by $\sim 4 \%$ modern carbon) at some point between time of assay at SSR and GrN and more recent measurements at Glasgow. 
Considered along with "check" samples reported later in the text, replicate measurement and accumulated NBS oxalic acid standard samples, samples GU-656-661 imply satisfactorily accurate measurement.

\section{REPLICATE ANALYSIS STANDARDIZATION}

Two replicate analysis "standards" were adopted for this study. A full description of relevant procedures may be found in Campbell (1977).

\section{Replicate Series 1}

Bulk homogenized wood (Pinus) from Ynyslas submerged forest exposure $\left(52^{\circ} 29^{\prime} \mathrm{N}, 4^{\circ} 4^{\prime} \mathrm{W}\right)$. Sample also subjected to sequential growthincrement analysis (see GU-711, -718, below). Coll by A Heyworth.

\begin{tabular}{lcc}
\hline $\begin{array}{c}\text { Sample } \\
\text { no. }\end{array}$ & Radiocarbon age & $\delta^{13} \mathrm{C} \%$ \\
\hline GU-669 & $4825 \pm 52$ & -24.0 \\
GU-670 & $5012 \pm 41$ & -24.4 \\
GU-671 & $4094 \pm 39$ & -24.2 \\
GU-672 & $5004 \pm 39$ & -23.7 \\
GU-673 & $4819 \pm 45$ & -24.1 \\
GU-674 & $5003 \pm 37$ & -24.2 \\
GU-675 & $4823 \pm 42$ & -23.8 \\
GU-676 & $4791 \pm 40$ & -24.1 \\
GU-677 & $4752 \pm 33$ & -24.0 \\
GU-678 & $4930 \pm 37$ & -23.9 \\
GU-679 & $4873 \pm 33$ & -23.7 \\
GU-680 & $4765 \pm 35$ & -23.9 \\
GU-681 & $4863 \pm 36$ & -23.7 \\
GU-682 & $4899 \pm 34$ & -23.7 \\
GU-683 & $4770 \pm 37$ & -23.9 \\
GU-684 & $4866 \pm 35$ & -23.8 \\
GU-685 & $4747 \pm 42$ & -23.9 \\
GU-686 & $4887 \pm 30$ & -24.0 \\
GU-687 & $4771 \pm 35$ & -24.0 \\
GU-688 & $4968 \pm 29$ & -24.1 \\
GU-689 & $4788 \pm 28$ & -24.4 \\
GU-690 & $4933 \pm 29$ & -26.1 \\
GU-691 & $4839 \pm 28$ & -23.9 \\
GU-692 & $4739 \pm 33$ & -24.2 \\
\hline
\end{tabular}

Comment: sample of replicate standard analyzed by SRR, $4926 \pm 52$ (Harkness, written commun).

\section{Replicate Series 2}

Rice grain.

\begin{tabular}{lccc}
\hline $\begin{array}{l}\text { Sample } \\
\text { no. }\end{array}$ & $\delta^{14} \mathrm{C} \%$ co & $\delta^{13} \mathrm{C} \%$ C \\
\hline GU-693 & $416.44 \pm 3.41$ & -23.9 & $413.32 \pm 3.42$ \\
GU-694 & $397.40 \pm 3.36$ & -24.0 & $394.61 \pm 3.36$ \\
GU-695 & $415.39 \pm 3.36$ & -24.3 & $413.41 \pm 3.36$ \\
GU-696 & $418.22 \pm 3.51$ & -25.4 & $419.36 \pm 3.50$ \\
GU-697 & $401.96 \pm 3.42$ & -25.0 & $401.96 \pm 3.42$ \\
GU-698 & $422.33 \pm 3.60$ & -23.8 & $418.92 \pm 3.61$ \\
GU-699 & $404.38 \pm 3.42$ & -22.8 & $398.20 \pm 3.44$ \\
GU-700 & $391.52 \pm 3.63$ & -24.1 & $389.02 \pm 3.64$ \\
GU-701 & $397.24 \pm 3.42$ & -24.0 & $394.45 \pm 3.43$ \\
GU-702 & $400.97 \pm 3.39$ & -23.8 & $397.43 \pm 3.40$ \\
\hline
\end{tabular}




\section{South Cadbury series}

Samples from site of Cadbury Castle, England (51 $\left.01^{\prime} \mathrm{N}, 2^{\circ} 32^{\prime} \mathrm{W}\right)$. Samples coll by L Alcock, Dept Archaeol, Univ Glasgow.

\section{GU-645. SC/K 659-i}

$$
\begin{array}{r}
1814 \pm \mathbf{3 1} \\
\delta^{13} C=-24.6 \%
\end{array}
$$

Wood charcoal. Twig remains from guard chamber, context $\mathrm{SC} / \mathrm{K}$ 659. Comment: sample material also analyzed as SRR-693, $1845 \pm 45$ (AD 105).

\section{GU-646. SC/K 659-ii}

$$
1961 \pm 27
$$

Wood charcoal assoc with large timbers from roof of guard chamber, context SC/K 659 .

\section{GU-647. SC/K 659-iii}

$1839 \pm 26$

$$
\delta^{13} C=-25.2 \% \text { o }
$$

Wood charcoal assoc with large timbers from roof of guard chamber, context SC/K 659 .

\section{GU-648. SC/K 659-iv}

$$
\begin{array}{r}
\mathbf{2 2 1 4} \pm \mathbf{4 3} \\
\delta^{13} C=-24.8 \%
\end{array}
$$

Wood charcoal assoc with large timbers from roof of guard chamber, context SC/K 659 .

\section{GU-649. SC/K 659-v}

$$
\begin{array}{r}
1949 \pm \mathbf{2 6} \\
\delta^{13} C=-24.1 \% \text { o }
\end{array}
$$

Carbonized grain from floor of guard chamber, context SC/K 659 . Comment: sample material also analyzed as SRR-691, $1776 \pm 50$ (AD 174).

\section{GU-650. SC/K 659-vi}

$$
\begin{array}{r}
1765 \pm 47 \\
\delta^{13} C=-24.3 \% 0
\end{array}
$$

Carbonized grain from floor of guard chamber, context SC/K 659 . Comment: sample material also analyzed as SRR-692, $1666 \pm 50$ (AD 284).

\section{GU-651. SC/K 747}

$1825 \pm 48$

$$
\delta^{13} C=-23.7 \%
$$

Wood charcoal assoc with structural timbers of gateway, context $\mathrm{SC} / \mathrm{K} 747$.

General Comment: South Cadbury series marks Roman sack of Cadbury Castle, an event placed in mid-first century AD, based on historico-archaeol evidence. Previous date on material from closely related level yielded age, AD $444 \pm 40$ (SRR-444), clearly at variance with archaeol evidence. This further study (Alcock et al, ms in preparation) shows improved concordance, although radiometric measurements still indicate more recent ages than theoretical prediction. 


\section{Los Tayos series}

Samples coll during 1976 British-Ecuadorian Expedition to Los Tayos Caves.

GU-652. OM-S6

$$
\begin{array}{r}
1012 \pm 66 \\
\delta^{1 s} C=-28.6 \%
\end{array}
$$

Wood charcoal assoc with "chimney-burial" pottery, Teniente Ortiz, Morona Santiago, Ecuador (3० $\left.3^{\prime} \mathrm{S}, 78^{\circ} 2^{\prime} \mathrm{W}\right)$.

\section{GU-653. OM-ST1-4}

Wood charcoal, Teniente Ortiz.

\section{GU-654. OM-ST1-5}

Wood charcoal, Teniente Ortiz.

GU-793. MS-LT-1

$$
\delta^{14} \mathbf{C}=\mathbf{2 . 5 8} \pm \mathbf{1 . 3 8} \% \text { o }
$$

Soil sample at $2.3 \mathrm{~m}$ depth in guano pile, main Los Tayos cave.

GU-792. Castle Point, Banffshire

$3107 \pm \mathbf{3 0 3}$

$\delta^{13} C=-25.1 \%$

Wood sample from early occupation site on Castle Point $\left(57^{\circ} 41^{\prime} 7^{\prime \prime}\right.$ $\left.\mathrm{N}, 2^{\circ} 17^{\prime} 26^{\prime \prime} \mathrm{W}\right)$.

$$
\text { IV. TREE RING SAMPLES }
$$

Past variations in natural radiocarbon content of the atmosphere were studied through ${ }^{14} \mathrm{C}$ assay of "floating" chronologies constructed from tree-ring records of preserved wood located in submerged forest sites along the west coast of England and Wales (Campbell, 1977). These forests provide an ideal source of material for dendrochronologic and radiocarbon studies. Individual exposures are mainly of oak, with some pine and, to a lesser extent, alder, birch, and yew. Growth patterns are extremely sensitive and individual rings are wide giving plentiful dating material and higher sampling frequency than in previous studies (Suess, 1970; 1976; Pearson et al, 1977). Dendrochronologic measurements are being made by A Heyworth, Univ College, Wales at Aberystwyth.

Preliminary dates on samples coll at several locations have indicated wide range of ages available in submerged forest exposures.

\section{GU-662. Clarach, Dyfed}

$5592 \pm 143$

Wood sec, Clarach-1 (Quercus) from submerged forest exposure $\left(52^{\circ}\right.$ $26^{\prime} \mathrm{N}, 4^{\circ} 4^{\prime} \mathrm{W}$ ). Coll by A Heyworth.

GU-663. Stolford, Somerset

$4443 \pm 199$

Wood sec Stolford-1 (Quercus) from submerged forest exposure $\left(51^{\circ}\right.$ $\left.12^{\prime} \mathrm{N}, 3^{\circ} 6^{\prime} \mathrm{W}\right)$. Coll by A Heyworth.

GU-664. Morecambe, Lancashire

$$
7544 \pm 306
$$

Wood sec Morecambe-1 (Betula) from submerged forest exposure $\left(54^{\circ} 04^{\prime} \mathrm{N}, 2^{\circ} 54^{\prime} \mathrm{W}\right)$. Coll by A Heyworth. 
GU-665. Llanaber, Gurynedd

Wood sec Llanaber-1 (Larix) from submerged forest exposure $\left(52^{\circ}\right.$ $\left.44^{\prime} \mathrm{N}, 4^{\circ} 4^{\prime} \mathrm{W}\right)$. Coll by A Heyworth.

GU-666. Alt-Mouth, Lancashire

$4351 \pm 46$

Wood sec Alt-Mouth-1 (Quercus) from submerged forest exposure $\left(53^{\circ} 32^{\prime} \mathrm{N}, 3^{\circ} 4^{\prime} \mathrm{W}\right)$. Coll by A Heyworth.

\section{GU-667. Newton, Powys}

$885 \pm 83$

Wood sec Newton-1 (Quercus) from gravel bed on River Severn $\left(52^{\circ}\right.$ $\left.31^{\prime} \mathrm{N}, 3^{\circ} 19^{\prime} \mathrm{W}\right)$.

GU-668. Rheidol, Dyfed

Wood sec Rheidol-1 (Quercus) from gravel bed on River Rheidol $\left(52^{\circ} 23^{\prime} \mathrm{N}, 3^{\circ} 58^{\prime} \mathrm{W}\right)$.

\section{Borth-1 tree-ring sequence, Borth, Dyfed series}

Wood sec Borth-1 (Pinus) comprising 90 growth rings. From submerged forest exposure $\left(52^{\circ} 29^{\prime} \mathrm{N}, 4^{\circ} 4^{\prime} \mathrm{W}\right)$. Coll by A Heyworth.

\begin{tabular}{lccc}
\hline $\begin{array}{c}\text { Sample } \\
\text { no. }\end{array}$ & $\begin{array}{c}\text { Growth } \\
\text { rings }\end{array}$ & $\begin{array}{c}\text { Radiocarbon } \\
\text { age }\end{array}$ & $\delta^{13} \mathrm{C}_{\% o}$ \\
\hline GU-703 & $0-20$ & $5167 \pm 42$ & -24.1 \\
GU-704 & $21-30$ & $5249 \pm 36$ & -24.1 \\
GU-705 & $31-40$ & $5277 \pm 37$ & -24.3 \\
GU-706 & $41-50$ & $5540 \pm 37$ & -23.9 \\
GU-707 & $51-60$ & $5240 \pm 36$ & -23.9 \\
GU-708 & $61-70$ & $5314 \pm 40$ & -23.9 \\
GU-709 & $71-80$ & $5124 \pm 36$ & -23.6 \\
GU-710 & $81-90$ & $5174 \pm 84$ & -23.9 \\
\hline
\end{tabular}

\section{Borth-4 tree-ring sequence, Borth, Dyfed series}

Wood sec Borth-4 (Quercus) comprising 155 growth rings. From submerged forest exposure $\left(52^{\circ} 29^{\prime} \mathrm{N}, 4^{\circ} 4^{\prime} \mathrm{W}\right)$. Coll by A Heyworth.

\begin{tabular}{lccc}
\hline $\begin{array}{l}\text { Sample } \\
\text { no. }\end{array}$ & $\begin{array}{c}\text { Growth } \\
\text { rings }\end{array}$ & $\begin{array}{c}\text { Radiocarbon } \\
\text { age }\end{array}$ & $\delta^{13} \mathrm{C}_{\%}$ \\
\hline GU-742 & $-15-+10$ & $3917 \pm 84$ & -26.1 \\
GU-743 & $11-30$ & $3781 \pm 95$ & -25.7 \\
GU-744 & $31-40$ & $3783 \pm 58$ & -27.0 \\
GU-745 & $41-50$ & $4027 \pm 35$ & -27.1 \\
GU-746 & $51-60$ & $3798 \pm 30$ & -25.5 \\
GU-747 & $61-70$ & $3832 \pm 31$ & -24.1 \\
GU-748 & $71-80$ & $3705 \pm 37$ & -24.8 \\
GU-749 & $81-90$ & $3751 \pm 61$ & -26.1 \\
GU-750 & $91-100$ & $3835 \pm 29$ & -24.5 \\
GU-75I & $101-110$ & $3838 \pm 46$ & -24.9 \\
GU-752 & $111-120$ & $3856 \pm 33$ & -24.7 \\
GU-753 & $121-130$ & $3812 \pm 31$ & -24.6 \\
GU-754 & $131-140$ & $3828 \pm 32$ & -24.5 \\
\hline
\end{tabular}

Comment: GU-746 was also analyzed by SRR, $3796 \pm 51$ (Harkness, written commun). 


\section{Borth-6 tree-ring sequence, Borth, Dyfed series}

Wood sec Borth-6 (Quercus) comprising 270 growth rings. From submerged forest exposure $\left(52^{\circ} 29^{\prime} \mathrm{N}, 4^{\circ} 4^{\prime} \mathrm{W}\right)$. Coll by A Heyworth.

\begin{tabular}{lccc}
\hline $\begin{array}{c}\text { Sample } \\
\text { no. }\end{array}$ & $\begin{array}{c}\text { Growth } \\
\text { rings }\end{array}$ & $\begin{array}{c}\text { Radiocarbon } \\
\text { age }\end{array}$ & $\delta^{13} \mathrm{C} / \%$ o \\
\hline GU-719 & $1-10$ & $4419 \pm 49$ & -25.7 \\
GU-720 & $11-22$ & $4284 \pm 47$ & -25.3 \\
GU-721 & $23-40$ & $4029 \pm 104$ & -25.0 \\
GU-722 & $41-69$ & $4052 \pm 35$ & -24.2 \\
GU-723 & $70-80$ & $4219 \pm 46$ & -24.1 \\
GU-724 & $81-90$ & $3958 \pm 36$ & -25.2 \\
GU-725 & $91-100$ & $3825 \pm 69$ & -26.1 \\
GU-726 & $101-112$ & $3762 \pm 54$ & -26.1 \\
GU-727 & $113-120$ & $3968 \pm 40$ & -23.1 \\
GU-728 & $121-130$ & $4872 \pm 42$ & -24.9 \\
GU-729 & $131-140$ & $3960 \pm 46$ & -25.4 \\
GU-730 & $141-150$ & $3738 \pm 43$ & -24.3 \\
GU-731 & $4120 \pm 45$ & -25.7 \\
GU-732 & $151-160$ & $4189 \pm 87$ & -25.3 \\
GU-733 & $161-170$ & $3961 \pm 47$ & -24.4 \\
GU-734 & $171-180$ & $4006 \pm 33$ & -24.0 \\
GU-735 & $181-190$ & $3984 \pm 60$ & -24.0 \\
GU-736 & $191-200$ & $3999 \pm 26$ & -24.2 \\
GU-737 & $201-210$ & $4198 \pm 44$ & -24.3 \\
GU-738 & $211-220$ & $4034 \pm 45$ & -24.3 \\
GU-739 & $221-230$ & $4068 \pm 45$ & -25.7 \\
GU-740 & $231-240$ & $3957 \pm 64$ & -25.0 \\
GU-741 & $241-250$ & & -24.0 \\
\hline
\end{tabular}

Comment: GU-730,-731 were also analyzed by SSR, yielding respective ages, $4016 \pm 53 ; 3958 \pm 52$ (Harkness, written commun).

\section{Ynyslas-1 tree-ring sequence, Ynyslas, Dyfed series}

Wood sec Ynyslas-1 (Pinus) comprising 80 growth rings. From submerged forest exposure $\left(52^{\circ} 29^{\prime} 30^{\prime \prime} \mathrm{N}, 4^{\circ} 4^{\prime} \mathrm{W}\right)$. Coll by A Heyworth.

\begin{tabular}{lccc}
\hline $\begin{array}{c}\text { Sample } \\
\text { no. }\end{array}$ & $\begin{array}{c}\text { Growth } \\
\text { rings }\end{array}$ & $\begin{array}{c}\text { Radiocarbon } \\
\text { age }\end{array}$ & $\delta^{13} \mathrm{C} /{ }^{\circ}$ \\
\hline GU-711 & $-10-+10$ & $5106 \pm 48$ & -24.5 \\
GU-712 & $0-10$ & $4935 \pm 89$ & -24.7 \\
GU-713 & $11-20$ & $5009 \pm 52$ & -23.9 \\
GU-714 & $21-30$ & $4974 \pm 39$ & -24.1 \\
GU -715 & $31-40$ & $4946 \pm 46$ & -24.3 \\
GU-716 & $41-50$ & $5035 \pm 46$ & -24.2 \\
GU-717 & $51-60$ & $4924 \pm 40$ & -23.6 \\
GU-718 & $61-70$ & $4930 \pm 48$ & -23.3 \\
\hline
\end{tabular}

\section{Stolford-4 tree-ring sequence, Stolford, Somerset series}

Wood sec Stolford-4 (Quercus) comprising 80 growth rings. From submerged forest exposure $\left(51^{\circ} 12^{\prime} \mathrm{N}, 3^{\circ} 6^{\prime} \mathrm{W}\right)$. Coll by A Heyworth. 


\begin{tabular}{cccc}
\hline $\begin{array}{c}\text { Sample } \\
\text { no. }\end{array}$ & $\begin{array}{c}\text { Growth } \\
\text { rings }\end{array}$ & $\begin{array}{c}\text { Radiocarbon } \\
\text { age }\end{array}$ & $\delta^{13} \mathrm{C}_{\% 0}$ \\
\hline GU-755 & $1-10$ & $4733 \pm 34$ & -24.1 \\
GU-756 & $11-20$ & $4803 \pm 36$ & -24.0 \\
GU-757 & $21-30$ & $4718 \pm 31$ & -24.4 \\
GU-758 & $31-40$ & $4645 \pm 41$ & -24.2 \\
GU-759 & $41-50$ & $4490 \pm 47$ & -23.9 \\
GU-760 & $51-60$ & $4715 \pm 31$ & -24.1 \\
GU-761 & $61-70$ & $4778 \pm 42$ & -23.1 \\
GU-762 & $71-80$ & $4707 \pm 32$ & -22.8 \\
\hline
\end{tabular}

\section{Stolford-5 tree-ring sequence, Stolford, Somerset series}

Wood sec Stolford-5 (Quercus) comprising 302 growth rings. From submerged forest exposure $\left(51^{\circ} 12^{\prime} \mathrm{N}, 3^{\circ} 6^{\prime} \mathrm{W}\right)$. Coll by A Heyworth.

\begin{tabular}{lccc}
\hline $\begin{array}{c}\text { Sample } \\
\text { no. }\end{array}$ & $\begin{array}{c}\text { Growth } \\
\text { rings }\end{array}$ & $\begin{array}{c}\text { Radiocarbon } \\
\text { age }\end{array}$ & $\delta^{13} \mathrm{C}^{\circ}$ \\
\hline GU-763 & $-12-+10$ & $5398 \pm 79$ & -23.9 \\
GU-764 & $11-20$ & $5298 \pm 66$ & -23.8 \\
GU-765 & $21-30$ & $5201 \pm 55$ & -23.6 \\
GU-766 & $31-40$ & $5285 \pm 45$ & -23.6 \\
GU-767 & $41-50$ & $5399 \pm 40$ & -23.3 \\
GU-768 & $51-60$ & $5269 \pm 40$ & -23.1 \\
GU-769 & $61-70$ & $5258 \pm 60$ & -23.2 \\
GU-770 & $71-80$ & $5186 \pm 45$ & -22.9 \\
GU-771 & $81-90$ & $5382 \pm 43$ & -23.0 \\
GU-772 & $91-100$ & $5377 \pm 41$ & -23.4 \\
GU-773 & $101-110$ & $5220 \pm 39$ & -23.6 \\
GU-774 & $111-120$ & $5169 \pm 53$ & -23.9 \\
GU-775 & $121-130$ & $5204 \pm 72$ & -23.7 \\
GU-776 & $131-140$ & $5094 \pm 51$ & -23.4 \\
GU-777 & $141-150$ & $5092 \pm 71$ & -24.0 \\
GU-778 & $151-160$ & $4840 \pm 127$ & -23.8 \\
GU-779 & $161-170$ & $5020 \pm 104$ & -23.5 \\
GU-780 & $171-180$ & $5039 \pm 61$ & -24.1 \\
GU-781 & $181-190$ & $5092 \pm 67$ & -23.7 \\
GU-782 & $191-200$ & $5007 \pm 61$ & -23.6 \\
GU-784 & $211-220$ & $5015 \pm 68$ & -23.6 \\
GU-785 & $221-230$ & $4844 \pm 97$ & -23.4 \\
GU-786 & $231-240$ & $4958 \pm 54$ & -23.8 \\
GU-787 & $241-250$ & $4775 \pm 67$ & -23.8 \\
GU-788 & $251-260$ & $4908 \pm 68$ & -24.3 \\
GU-789 & $261-270$ & & -29.9 \\
GU-790 & $271-280$ & -23.4 \\
GU-791 & $281-290$ & -24.7 \\
\hline
\end{tabular}

General Comment: results of sequential growth-increment analyses clearly indicate that short-term fluctuations in natural radiocarbon content of the atmosphere have occurred over the past millennia. Variations of 2 to $3 \%$ over several decades are comparable with those reported by Campbell et al (1978) from similar measurements on a "floating" tree-ring chronology dating from early 3 rd millennium BP (radiocarbon years). It is firmly believed that detection of these variations has been facilitated by high sampling frequency employed in this study. 


\section{REFERENCES}

Campbell, J A, 1977, Past variations in natural radiocarbon as recorded in UK wood: PhD thesis, Univ Glasgow.

Campbell, J A, Baxter, M S, and Harkness, D D, 1978, Radiocarbon measurements on a floating tree-ring chronology from north-east Scotland: Archaeometry, v 20, p 33-38.

Pearson, G W, Pilcher, J R, Baillie, M G L, and Hillam, J, 1978, Absolute radiocarbon dating using a low altitude European tree-ring calibration: Nature, v 270, p 25-28.

Suess, H E, 1970, Bristlecone pine calibration of the radiocarbon time-scale $5200 \mathrm{BC}$ to the present, in: Olsson, I U (ed), Radiocarbon variations and absolute chronology, Nobel symposium 12th, Proc, Uppsala, 1969, p 303-312, New York, John Wiley. 1976, 9th internatl radiocarbon dating conf, Proc, Los Angeles, in press. 